\title{
THE NUMERICAL SOLUTION OF HAMMERSTEIN EQUATIONS BY A METHOD BASED ON POLYNOMIAL COLLOCATION
}

\author{
SUNIL KUMAR ${ }^{1}$
}

(Received 22 January 1988; revised 15 March 1989)

\begin{abstract}
In recent papers we have considered the numerical solution of the Hammerstein equation

$$
y(t)=f(t)+\int_{-1}^{1} k(t, s) g(s, y(s)) d s, \quad t \in[-1,1],
$$

by a method which first applies the standard collocation procedure to an equivalent equation for $z(t):=g(t, y(t))$, and then obtains an approximation to $y$ by use of the equation

$$
y(t)=f(t)+\int_{-1}^{1} k(t, s) z(s) d s, \quad t \in[-1,1] .
$$

In this paper we approximate $z$ by a polynomial $z_{n}$ of degree $\leq n-1$, with coefficients determined by collocation at the zeros of the $n$th degree Chebyshev polynomial of the first kind. We then define the approximation to $y$ to be

$$
y_{n}(t):=f(t)+\int_{-1}^{1} k(t, s) z_{n}(s) d s, \quad t \in[-1,1],
$$

and establish that, under suitable conditions, $\lim _{n \rightarrow \infty} y_{n}(t)=y(t)$, uniformly in $t$.
\end{abstract}

\section{Introduction}

This paper is concerned with the Kumar and Sloan method [10] for the numerical solution of the Hammerstein equation

$$
y(t)=f(t)+\int_{-1}^{1} k(t, s) g(s, y(s)) d s, \quad t \in[-1,1]
$$

'School of Mathematics, The University of New South Wales, Sydney, NSW 2033, Australia. (C) Copyright Australian Mathematical Society 1990, Serial-fee code 0334-2700/90 
where $f, k$, and $g$ are known functions, with $g(s, v)$ nonlinear in $v$, and $y$ is the solution to be determined.

The Kumar and Sloan method is a collocation method applied not to equation (1.1), but rather to an equivalent equation (see (1.4)) for the function $z$ defined by

$$
z(t):=g(t, y(t)), \quad t \in[-1,1]
$$

The desired approximation to the solution $y$ of $(1.1)$ is then obtained by the use of the (exact) equation

$$
y(t)=f(t)+\int_{-1}^{1} k(t, s) z(s) d s, \quad t \in[-1,1] .
$$

Kumar and Sloan established that, under suitable conditions, the approximation to $y$ converges to the exact solution at a rate at least equal to that of the best approximation to $z$ from the space in which the collocation solution is sought. For the particular case where the collocation approximation to $z$ is sought in certain piecewise polynomial function spaces, Kumar [9] showed that the approximation to $y$ may exhibit (global) superconvergence, that is, it may converge to $y$ at a faster rate than the collocation approximation does to $z$.

In this paper we approximate $z$ by a polynomial $z_{n}$ of degree $\leq n-1$, with coefficients determined by collocating the equation

$$
z(t)=g\left(t, f(t)+\int_{-1}^{1} k(t, s) z(s) d s\right), \quad t \in[-1,1],
$$

at the $n$ zeros $\left\{\tau_{n i}\right\}_{i=1}^{n}$ of the $n$th degree Chebyshev polynomial of the first kind. Thus $z_{n}$ is that polynomial of degree $\leq n-1$ which satisfies the $n$ nonlinear equations:

$$
z_{n}\left(\tau_{n i}\right)=g\left(\tau_{n i}, f\left(\tau_{n i}\right)+\int_{-1}^{1} k\left(\tau_{n i}, s\right) z_{n}(s) d s\right), \quad i=1, \ldots, n .
$$

We then take

$$
y_{n}(t):=f(t)+\int_{-1}^{1} k(t, s) z_{n}(s) d s, \quad t \in[-1,1],
$$

as the approximation to $y$, and establish that, under suitable conditions,

$$
\lim _{n \rightarrow \infty} y_{n}(t)=y(t)
$$

uniformly in $t$. We also establish a rate for the convergence of $y_{n}$ to $y$ that is fast enough to yield, in favourable circumstances, impressive accuracy even for polynomials of low degree (for an example, see Section 6). Obviously, low degree polynomials imply small nonlinear systems, something which is highly 
desirable in practical computations. We remark that the above convergence result is the analogue of that proved by Sloan and Burn [15] for the iterated (polynomial) collocation solution of (linear) integral equations of the second kind.

It should be noted that the major difference between the present analysis and that in [10] is the fact that for polynomial interpolation, uniform convergence of the interpolants cannot be guaranteed for every continuous function, regardless of the choice of the interpolation points. Hence, the present analysis is based on a mean-convergence property (see (3.5)) of polynomial interpolation.

\section{Basic assumptions and operators}

The theoretical analysis of (1.1) will be carried out in the Banach space $C=C[-1,1]$ of continuous, real-valued functions on $[-1,1]$. This space is equipped with the uniform norm

$$
\|x\|_{\infty}=\sup _{-1 \leq t \leq 1}|x(t)|, \quad x \in C .
$$

The following assumptions are made on the functions $f, k$, and $g$ in (1.1):

A1: $f \in C$;

A2: the kernel $k$ satisfies

$$
\sup _{-1 \leq i \leq 1} \int_{-1}^{1}|k(t, s)|^{p} d s<\infty
$$

and

$$
\lim _{t \rightarrow t^{\prime}} \int_{-1}^{1}\left|k(t, s)-k\left(t^{\prime}, s\right)\right|^{p} d s=0, \quad t^{\prime} \in[-1,1],
$$

for some $p>1$;

A3: the function $g(t, v)$ is defined and continuous on $[-1,1] \times \mathbf{R}$;

A4: the partial derivative $g_{v}(t, v):=\frac{\partial}{\partial v} g(t, v)$ exists and is continuous on $[-1,1] \times \mathbf{R}$.

With $p$ as in assumption $\mathrm{A} 2$, let $q$ be the number given by

$$
\frac{1}{q}+\frac{1}{p}=1
$$

Furthermore, let $L_{q}=L_{q}(-1,1)$ denote the Banach space of measurable, real-valued functions on $(-1,1)$ which have integrable $q$ th power. It then follows from the results of [7] that, under assumption A2, the linear integral operator $K$, defined by

$$
(K w)(t):=\int_{-1}^{1} k(t, s) w(s) d s, \quad t \in[-1,1], w \in L_{q},
$$


is a compact operator from $L_{q}$ to $C$, and hence also from $C \subset L_{q}$ to $C$. Being both compact and linear, it is necessarily (see [8], p. 244) completely continuous. It is also worth mentioning that [7] gives certain tests for deciding whether A2 holds in a given case. In particular, we note that A2 is satisfied for every continuous kernel, and for some weakly singular kernels.

We define another completely continuous operator $T: L_{q} \rightarrow C$ by

$$
T(w)(t):=f(t)+(K w)(t), \quad t \in[-1,1], w \in L_{q},
$$

and a continuous, bounded operator $G: C \rightarrow C$ by

$$
G(x)(t):=g(t, x(t)), \quad t \in[-1,1], x \in C .
$$

With the above notation, the integral equations (1.1) and (1.4) may be written in operator form as

$$
y=T G(y), \quad y \in C,
$$

and

$$
z=G T(z), \quad z \in L_{q},
$$

respectively. The two equations are equivalent in the sense of the following lemma.

LEMMA 1 [10]. The sets

$$
\boldsymbol{\Theta}_{T G}:=\{y \in C: T G(y)=y\}
$$

and

$$
\Theta_{G T}:=\left\{z \in L_{q}: G T(z)=z\right\}
$$

are in one-to-one correspondence. Specifically, $G$ is a one-to-one operator from $\boldsymbol{\theta}_{T G}$ onto $\boldsymbol{\theta}_{G T}$, with inverse $T$.

For later use we note that, under assumptions $\mathrm{Al}$ to $\mathrm{A} 4$, the operator $G T$ is continuously Fréchet differentiable on $L_{q}$. Its Fréchet derivative at $z \in L_{q}$ is the completely continuous linear operator $(G T)^{\prime}(z)$ given by

$$
\left[(G T)^{\prime}(z) w\right](t)=g_{v}(t, f(t)+(K z)(t))(K w)(t), \quad t \in[-1,1], w \in L_{q} .
$$

\section{The polynomial interpolation operator}

For $w \in C$, let $P_{n} w$ denote the unique polynomial of degree $\leq n-1$ that satisfies

$$
\left(P_{n} w\right)\left(\tau_{n j}\right)=w\left(\tau_{n j}\right), \quad j=1, \ldots, n
$$


where

$$
\tau_{n j}=\cos \frac{2 j-1}{2 n} \pi, \quad j=1, \ldots, n .
$$

The points $\left\{\tau_{n j}\right\}_{j=1}^{n}$ are known as the classical Chebyshev points, and are the zeros of the $n$th degree Chebyshev polynomial of the first kind $T_{n}$, defined by $T_{n}(\cos \theta):=\cos n \theta$. Recall that polynomial interpolation based on the points (3.2) is relatively well behaved [14, Section 1.3] compared to that based on equally spaced points $[5, \mathrm{p} .78]$.

In the Lagrange form $P_{n} w$ is

$$
\left(P_{n} w\right)(t)=\sum_{j=1}^{n} w\left(\tau_{n j}\right) l_{n j}(t), \quad t \in[-1,1],
$$

where $l_{n j}$ (a 'fundamental Lagrange polynomial') is the unique polynomial of degree $\leq n-1$ that satisfies

$$
l_{n j}\left(\tau_{n i}\right)=\delta_{i j}, \quad 1 \leq i, j \leq n,
$$

where $\delta_{i j}$ is the Kronecker delta. Clearly, $P_{n}$ is a linear operator on $C$, with the property $P_{n}^{2}=P_{n}$. It is therefore a projection, whose range is $P_{n}$, the set of all polynomials of degree $\leq n-1$. Furthermore, $P_{n}$ is a bounded operator on $C$, with

$$
\left\|P_{n}\right\|=\sup _{-1 \leq t \leq 1} \sum_{j=1}^{n}\left|l_{n j}(t)\right| .
$$

Since $P_{n}$ is the interpolatory operator defined by (3.3), and the collocation points are given by $(3.2)$, it follows $[1,2,6,11,12]$ that

$$
\lim _{n \rightarrow \infty} \int_{-1}^{1}\left|\left(P_{n} w\right)(s)-w(s)\right|^{q}\left(1-s^{2}\right)^{-1 / 2} d s=0
$$

for every $w \in C$, and for every real number $q$ in the range $0<q<\infty$. As in Section 2, we choose $q$ to be the number given by (2.1).

Let $\rho$ be the weight function

$$
\rho(s):=\left(1-s^{2}\right)^{-1 / 2}, \quad s \in(-1,1),
$$

and let $L_{q, \rho}=L_{q, \rho}(-1,1)$ be the space of functions $x \in L_{q}$ for which the weighted $L_{q}$ norm defined by

$$
\|x\|_{q, \rho}:=\left(\int_{-1}^{1}|x(s)|^{q} \rho(s) d s\right)^{1 / q}
$$

is finite. In terms of this norm, (3.4) may be written as

$$
\lim _{n \rightarrow \infty}\left\|P_{n} w-w\right\|_{q, \rho}=0
$$


from which it follows that

$$
\sup _{n}\left\|P_{n} w\right\|_{q, \rho}<\infty
$$

for all $w \in C$. Thus, if $P_{n}$ is considered as a linear operator from the space $C$ to the space $L_{q, p}$ then, from the Banach-Steinhaus theorem [8, p. 203], it follows that there exists a constant $c_{1}>0$, which depends only on $q$, such inai

$$
\left\|P_{n} x\right\|_{q, \rho} \leq c_{1}\|x\|_{\infty}, \quad x \in C .
$$

REMARK 1. The analysis given in this paper will hold for any set of collocation points and any weight function $\rho$, provided that (3.5) is satisfied for the $q$ fixed by (2.1), and that $1 / \rho$ is bounded. In the case of Jacobi weight functions and interpolation at zeros of the associated Jacobi polynomials, the values of $q$ for which (3.5) holds are easily obtained from [1, 2]. It should be pointed out that while the Jacobi case considered here permits $q$ in (3.5) (ignoring (2.1)) to be any positive number, the other cases are less generous with the values $q$ may assume. For example, $0<q<4$ for the Legendre points $(\rho=1)$.

\section{Convergence results}

The mean-convergence property (3.5) suggests that an appropriate space for the analysis of (1.4) is the Banach space $L_{q, \rho} \subset L_{q}$ equipped with the norm $\|\cdot\|_{q, \rho}$. Then the theory of [10] goes through in an obvious manner for the equations

and

$$
z=G T(z), \quad z \in L_{q, \rho},
$$

$$
z_{n}=P_{n} G T\left(z_{n}\right), \quad z_{n} \in \mathbf{P}_{n} \subset L_{q, \rho} .
$$

(Note that (4.2) is the collocation equation (1.5) written in operator form.)

Below we give results analogous to those in [10, Section 4].

Theorem 1. Let $y^{*} \in C$ be a geometrically isolated solution of (2.2), and let $z^{*}$ be the corresponding solution of (4.1). Suppose A1 to A3 hold.

(i) If $y^{*}$ has a nonzero index, then there exists an $n_{0}$ such that for $n \geq n_{0}$, (4.2) has a solution $z_{n} \in \mathbf{P}_{n}$ satisfying

$$
\left\|z^{*}-z_{n}\right\|_{q, \rho} \rightarrow 0 \text { as } n \rightarrow \infty \text {. }
$$

(ii) Suppose A4 holds, and that 1 is not an eigenvalue of the linear operator $(G T)^{\prime}\left(z^{*}\right)$. Then there exists a neighbourhood of $z^{*}$ and an $n_{1}$ such that for $n \geq n_{1}$ a solution $z_{n}$ of (4.2) is unique in that neighbourhood, and

$$
c_{2}\left\|z^{*}-P_{n} z^{*}\right\|_{q, \rho} \leq\left\|z^{*}-z_{n}\right\|_{q, \rho} \leq c_{3}\left\|z^{*}-P_{n} z^{*}\right\|_{q, \rho},
$$

where $c_{2}, c_{3}>0$ are independent of $n$. 
COROLlaRY 1. Under the conditions in Theorem 1 (ii) there exists a constant $c_{4}>0$ such that

$$
\left\|z^{*}-z_{n}\right\|_{q, \rho} \leq c_{4} \inf _{\varphi \in \mathbf{P}_{n}}\left\|z^{*}-\varphi\right\|_{\infty} .
$$

Proof. For any $\varphi \in \mathbf{P}_{n}$,

$$
\begin{aligned}
\left\|z^{*}-P_{n} z^{*}\right\|_{q, \rho} & =\left\|\left(z^{*}-\varphi\right)-P_{n}\left(z^{*}-\varphi\right)\right\|_{q, \rho} \\
& \leq\left\|z^{*}-\varphi\right\|_{q, \rho}+\left\|P_{n}\left(z^{*}-\varphi\right)\right\|_{q, \rho} \\
& \leq\left[\int_{-1}^{1}\left|z^{*}(s)-\varphi(s)\right|^{q} \rho(s) d s\right]^{1 / q}+c_{1}\left\|z^{*}-\varphi\right\|_{\infty} \\
& \leq\left(\left[\int_{-1}^{1} \rho(s) d s\right]^{1 / q}+c_{1}\right)\left\|z^{*}-\varphi\right\|_{\infty},
\end{aligned}
$$

where the second last step follows from the fact that $P_{n}$ is uniformly bounded as an operator from $C$ to $L_{q, \rho}$.

It should be noted that the quantity

$$
\inf _{\varphi \in \mathbf{P}_{n}}\left\|z^{*}-\varphi\right\|_{\infty}
$$

approaches zero as $n \rightarrow \infty$ because of the Weierstrass approximation theorem [ 5 , Theorem 6.1.1], and may be bounded above by use of the Jackson theorems [4, p. 147].

Theorem 2. Let $y^{*} \in C$ be a geometrically isolated solution of (2.2), and let $z^{*}$ be the corresponding solution of (4.1). Suppose A1 and A3 hold.

(i) If $y^{*}$ has a nonzero index, then with $z_{n}$ as in Theorem $1(\mathrm{i})$, and $n \geq n_{0}$, (1.6) defines an approximation $y_{n} \in C$ satisfying

$$
\left\|y^{*}-y_{n}\right\|_{\infty} \rightarrow 0 \text { as } n \rightarrow \infty \text {. }
$$

(ii) Suppose A4 holds, and that 1 is not an eigenvalue of the linear operator $(G T)^{\prime}\left(z^{*}\right)$. Then for $n \geq n_{1}$ the approximation $y_{n}$ given by (1.6), with $z_{n}$ as in Theorem 1(ii), satisfies

$$
\left\|y^{*}-y_{n}\right\|_{\infty} \leq c_{5} \inf _{\varphi \in \mathbf{P}_{n}}\left\|z^{*}-\varphi\right\|_{\infty}
$$

where $c_{5}>0$ is independent of $n$.

Proof. (i) Since $T$ maps $L_{q, \rho}$ to $C$, it follows immediately from the definition $y_{n}:=T\left(z_{n}\right)$ that $y_{n} \in C$. Now from Lemma 1 ,

$$
y^{*}=T\left(z^{*}\right)=f+K z^{*},
$$


and hence

$$
\begin{aligned}
\mid y^{*}(t) & -y_{n}(t)|=| \int_{-1}^{1} k(t, s)\left[z^{*}(s)-z_{n}(s)\right] d s \mid \\
& \leq \int_{-1}^{1}|k(t, s) / \rho(s)|\left|z^{*}(s)-z_{n}(s)\right| \rho(s) d s \\
& \leq\left[\int_{-1}^{1}|k(t, s) / \rho(s)|^{p} \rho(s) d s\right]^{1 / p} \times\left[\int_{-1}^{1}\left|z^{*}(s)-z_{n}(s)\right|^{q} \rho(s) d s\right]^{1 / q},
\end{aligned}
$$

where in the last step we have used the Hölder inequality for the distribution $\rho(s) d s$. Now for $p>1$,

$$
\sup _{-1 \leq s \leq 1}[\rho(s)]^{1-p}=1
$$

Therefore,

$$
\left|y^{*}(t)-y_{n}(t)\right| \leq\left[\int_{-1}^{1}|k(t, s)|^{p} d s\right]^{1 / p}\left\|z^{*}-z_{n}\right\|_{q, \rho},
$$

and hence

$$
\left\|y^{*}-y_{n}\right\|_{\infty} \leq c\left\|z^{*}-z_{n}\right\|_{q, \rho} \rightarrow 0 \quad \text { as } n \rightarrow \infty
$$

where

$$
c=\sup _{-1 \leq t \leq 1}\left[\int_{-1}^{1}|k(t, s)|^{p} d s\right]^{1 / p}<\infty .
$$

(ii) This follows similarly, with the aid of Corollary 1.

Thus $y_{n}$ converges uniformly to $y^{*}$ as $n \rightarrow \infty$.

\section{Implementation note}

In practice, it may be worthwhile expressing $z_{n}$ as a finite Chebyshev series of the form

$$
z_{n}(t)=\sum_{j=0}^{n-1} a_{n j} T_{j}(t), \quad t \in[-1,1],
$$

where $T_{j}$ is the $j$ th degree Chebyshev polynomial of the first kind. Then (1.5) yields a system of $n$ nonlinear equations

$$
\begin{aligned}
& \sum_{j=0}^{n-1} a_{n j} T_{j}\left(\tau_{n i}\right)=g \tau_{n i}, f\left(\tau_{n i}\right)+\sum_{j=0}^{n-1} a_{n j} \int_{-1}^{1} k\left(\tau_{n i}, s\right) T_{j}(s) d s, \\
& i=1, \ldots, n,
\end{aligned}
$$


for the $n$ coefficients $a_{n 0}, \ldots, a_{n, n-1}$. Once these coefficients have been computed, $y_{n}$ can be calculated from

$$
y_{n}(t)=f(t)+\sum_{j=0}^{n-1} a_{n j} \int_{-1}^{1} k(t, s) T_{j}(s) d s, \quad t \in[-1,1] .
$$

Clearly, the main practical difficulty lies in calculating the integrals

$$
K T_{j}(t)=\int_{-1}^{1} k(t, s) T_{j}(s) d s, \quad t \in[-1,1]
$$

which are required in both (5.2) and (5.3). It is here that the advantage of expressing $z_{n}$ as the finite Chebyshev series (5.1) appears: for many kernels of practical interest, the integrals $K T_{j}(t)$ (where $t$ has any fixed value) may be evaluated recursively [13], hence saving much of the computational work.

\section{A numerical example}

In this section the proposed method is used to solve an integral equation reformulation of the nonlinear two-point boundary-value problem

$$
y^{\prime \prime}(t)-\exp y(t)=0, \quad t \in(0,1) ; \quad y(0)=y(1)=0,
$$

which apparently is of some interest in magnetohydrodynamics [3, p. 41]. This problem has the unique solution

$$
y^{*}(t)=-\ln 2+2 \ln \left(c / \cos \left(\frac{1}{2} c\left(t-\frac{1}{2}\right)\right)\right), \quad t \in[0,1],
$$

where $c=1.3360556949 \ldots$ is the root of $c / \cos (c / 4)=\sqrt{2}$. It may be reformulated as the integral equation

$$
y(t)=\int_{0}^{1} k(t, s) \exp y(s) d s, \quad t \in[0,1]
$$

where

$$
k(t, s)= \begin{cases}-s(1-t), & s \leq t, \\ -t(1-s), & s>t,\end{cases}
$$

is the Green's function for the homogeneous problem

$$
y^{\prime \prime}(t)=0, \quad t \in(0,1) ; \quad y(0)=y(1)=0 .
$$

From the integral equation it follows that $y(t)$ is symmetric about $t=1 / 2$. Taking advantage of this symmetry, the solution $z^{*}(t)=\exp \left(y^{*}(t)\right)$ of the integral equation

$$
z(t)=\exp \left(\int_{0}^{1} k(t, s) z(s) d s\right), \quad t \in[0,1]
$$


was approximated by the Chebyshev series

$$
z_{2 m-1}(t)=\sum_{j=0}^{m-1} a_{2 m-1,2 j} T_{2 j}(2 t-1), \quad t \in[0,1],
$$

with the coefficients $a_{2 m-1,2 j}$ determined by collocation at the points

$$
\tau_{2 m-1, i}=\frac{1}{2}\left(1+\cos \frac{2 i-1}{4 m-2} \pi\right), \quad i=1, \ldots, m .
$$

Note that these points are just the classical Chebyshev points on the interval $[0,1]$ shifted linearly to the interval $[1 / 2,1]$. All required integrals were calculated exactly by Gaussian quadrature of appropriate precision.

The results, which are displayed in Table 1, show that very high accuracy is obtained even when the polynomials are of low degree. Note that the degree is $2 m-2$, and the size of the corresponding nonlinear system is $m$. It should be mentioned that the present problem has been solved in $[9,10]$ by the corresponding piecewise polynomial scheme. There very much larger nonlinear systems had to be solved to obtain accuracy of comparable order.

TABLE 1

\begin{tabular}{|cc|}
\hline$m$ & $\left\|y^{*}-y_{2 m-1}\right\|_{\infty}$ \\
\hline 2 & $5.27 \mathrm{E}-5$ \\
3 & $2.57 \mathrm{E}-7$ \\
4 & $1.47 \mathrm{E}-9$ \\
5 & $8.79 \mathrm{E}-12$ \\
6 & $7.97 \mathrm{E}-14$ \\
\hline
\end{tabular}

\section{References}

[1] R. Askey, "Mean convergence of orthogonal series and Lagrange interpolation," Acta Math. Acad. Sci. Hungar. 23 (1972) 71-85.

[2] R. Askey, "Summability of Jacobi series", Trans. Amer. Math. Soc. 179 (1973) 71-84.

[3] R. E. Bellman and R. E. Kalaba, Quasilinearization and nonlinear boundary-value problems (Elsevier, New York, 1965).

[4] E. W. Cheney, Introduction to approximation theory (McGraw-Hill, New York, 1966).

[5] P. J. Davis, Interpolation and approximation (Blaisdell, New York, 1963).

[6] P. Erdös and E. Feldheim, "Sur le mode de convergence pour l'interpolation de Lagrange”, C. R. Acad. Sci. Paris Sér. A-B 203 (1936) 913-915.

[7] 1. G. Graham and I. H. Sloan, "On the compactness of certain integral operators", J. Math. Anal. Appl. 68 (1979) 580-594. 
[8] L. V. Kantorovich and G. P. Akilov, Functional analysis (Pergamon Press, Oxford, 1982).

[9] S. Kumar, "Superconvergence of a collocation-type method for Hammerstein equations", IMA J. Numer. Anal. 7 (1987) 313-325.

[10] S. Kumar and I. H. Sloan, "A new collocation-type method for Hammerstein integral equations", Math. Comp. 48 (1987) 585-593.

[11] J. Marcinkiewicz, "Sur l'interpolation (1)", Studia Math. 6 (1936) 1-17.

[12] G. P. Nevai, "Lagrange interpolation at zeros of orthogonal polynomials", in Approximation theory II (eds. G. G. Lorentz, C. K. Chui and L. L. Schumaker), (Academic Press, New York, 1976), 163-201.

[13] R. Piessens and M. Branders, "Numerical solution of integral equations of mathematical physics, using Chebyshev polynomials”, J. Comput. Phys. 21 (1976) 178-196.

[14] T. J. Rivlin, The Chebyshev polynomials (Wiley, New York, 1974).

[15] I. H. Sloan and B. J. Burn, "Collocation with polynomials for integral equations of the second kind: a new approach to the theory", J. Integral Equations 1 (1979) 77-94. 